\title{
Características de los estomas, densidad e índice estomático en secuoya (Sequoia sempervirens) y su variación en diferentes plantaciones de Chile
}

\author{
Stomata Characteristics, stomatal density and stomatic index in redwood (Sequoia sempervirens) \\ and its variation in different plantations in Chile
}

\author{
Manuel Torala*, Adelina Manríquez $^{\mathrm{a}}$, Rafael Navarro-Cerrillo ${ }^{\mathrm{b}}$, Denise Tersi ${ }^{\mathrm{a}}$, Paulette Naulin ${ }^{\mathrm{a}}$ \\ *Autor de correspondencia: aUniversidad de Chile, Facultad de Ciencias Forestales y Conservación de la Naturaleza, casilla \\ 9206, Santiago, Chile, tel.: 56-2-9785898, mtoral@uchile.cl \\ bUniversidad de Córdoba, Departamento de Ingeniería Forestal, Córdoba, España.
}

\begin{abstract}
SUMMARY
Several authors suggest that the stomata of Sequoia sempervirens (redwood) may present morphological changes when this species grows in areas without the presence of crypto-precipitation. This work studied the characteristics of the stomata, density and stomata index in leaves of redwood in terms of precipitation level, soil, slope exposition and location in the forest stand. The anatomical study was made by diaphanization and staining techniques of the leaf samples. The main results of this work indicate that the sequoia stomata, in the populations studied, are smaller than those from natural populations, with an average length between 31.4 and $37.7 \mu \mathrm{m}$ and an average width between 12.6 and $14.9 \mu \mathrm{m}$; mainly found in the abaxial epidermis. Their density varied between 81.3 and 111.4 stomata $\mathrm{mm}^{-2}$, with an average stomatic index between 15.5 and 21.1 depending on the locality studied. These values are in ranks lower than those found for the species by other authors; indicating a certain degree of acclimation for the species in Chile. While there was significant variation among localities in Chile, changes in the stomatal complex were not so evident in response to the site environmental characteristics. However, in areas with more restraining climatic conditions (lower atmospheric humidity and precipitation), combined with edaphic conditions (soil with greater apparent density or less depth), stomata showed smaller and less dense, revealing a significant degree of phenotypic plasticity of the species for the character studied, which allows adaptation processes to contrasting environmental situations.
\end{abstract}

Key words: leaf morphology, cuticle, epidermis, Taxodiaceae.

\section{RESUMEN}

Varios autores sugieren que los estomas de secuoya (Sequoia sempervirens) pueden presentar cambios morfológicos cuando esta especie crece en áreas con ausencia de criptoprecipitación. En este trabajo se estudiaron las características de los estomas, densidad e índice estomático en hojas de secuoya en función del nivel de precipitación, suelo, exposición y ubicación del árbol en el rodal. El estudio anatómico se realizó mediante técnicas de diafanización y teñido de las muestras de hojas. Los principales resultados indican que los estomas de secuoya en las poblaciones estudiadas son pequeños en comparación con las poblaciones naturales, con longitud media entre 31,4 y $37,7 \mu \mathrm{m}$ y ancho medio entre 12,6 y $14,9 \mu \mathrm{m}$, y se presentan principalmente en la epidermis abaxial. Su densidad media varió entre 81,3 y 111,4 estomas $\mathrm{mm}^{-2}$, con un índice estomático medio entre 15,5 y 21,1 según la localidad estudiada. Estos valores están en los rangos inferiores a los encontrados para la especie por otros autores, lo que indica cierto grado de aclimatación para la especie en Chile. Si bien hubo variación significativa entre localidades de Chile, los cambios en el aparato estomático no fueron tan marcados en respuesta a las características ambientales de los sitios. Sin embargo, en localidades con condiciones climáticas más limitantes (menor humedad atmosférica y precipitación), en combinación con las condiciones edáficas (suelos con mayor densidad aparente o menor profundidad), mostraron estomas más pequeños y en menor densidad, revelando un importante grado de plasticidad fenotípica de la especie para el carácter evaluado, que permite procesos de aclimatación a situaciones ambientales contrastantes.

Palabras clave: morfología foliar, cutícula, epidermis, Taxodiaceae.

\section{INTRODUCCIÓN}

Sequoia sempervirens (D. Don) Endl. (secuoya) es una especie que se desarrolla naturalmente desde el extremo sur este del Estado de Oregón hasta el Condado de Monterrey en California (Benson 1979), formando una franja irregular de distribución en la costa oeste californiana. El área de distribución de la especie se caracteriza por un clima marítimo húmedo, la lluvia se concentra en invierno, con veranos secos y con fuerte presencia de neblinas, especialmente en verano. La secuoya presenta una gran capacidad de adaptación a la precipitación horizontal (Burgess y 
Dawson 2004), habiéndose observado que esta especie es capaz de obtener entre un $8 \%$ y un $34 \%$ del agua a partir de la criptoprecipitación (aporte adicional de agua por presencia de niebla) (Dawson 1998). La presencia de neblina en verano disminuye el estrés por sequía reduciendo la evapotranspiración y agregando humedad al suelo, lo que le permite a la secuoya crecer en estas condiciones.

Los estomas de secuoya han sido estudiados por varios autores (Srinivasan y Friis 1989, Chaturvedi 1993, Villar de Seoane 1998, Ma y Li 2002), habiéndose descrito la especie como hipoestomática, con estomas sólo en la epidermis abaxial (Chaturvedi 1993, Villar de Seoane 1998). En un estudio posterior, Ma et al. (2005) demostraron que el tamaño de los estomas en secuoya es similar al de otras coníferas y taxodiáceas. Las especies forestales que tienen áreas de distribución muy amplias, o bien se utilizan en plantaciones forestales en zonas con condiciones climáticas muy contrastadas con respecto a su área original (S. sempervirens, Pinus radiata D.Don), poseen estrategias de adaptación que les permiten sobrevivir y crecer en zonas ecológicamente diferenciadas (Poyatos et al. 2007). La hoja es el órgano de la planta más sensible a las condiciones ambientales, por lo cual refleja alteraciones morfológicas como consecuencia de los efectos de estrés (Trewavas 2003). Los mecanismos morfológicos y fisiológicos implicados en los procesos de adaptación-aclimatación frente al estrés operan a diferentes niveles de la planta, con el objetivo de asegurar la funcionalidad hídrica en las nuevas condiciones ambientales (Mencuccini 2003), siendo los estomas vitales para la homeóstasis de la planta. Estos procesos se han observado en distintas especies de coníferas, en particular en pinos (Pinus spp.) (DeLucia et al. 2000, Mencuccini y Bonosi 2001). La secuoya se ha utilizado ampliamente en programas forestales con carácter productor, tanto en América del Norte y del Sur, como en Asia y Europa, por lo que es factible pensar que la especie haya experimentado procesos de aclimatación en las localizaciones distintas a su área de distribución natural.

La especie fue introducida en Chile por Federico Albert en 1908 para estabilizar las dunas de Chanco, Constitución, y recomendada por éste como una especie promisoria para el desarrollo de plantaciones forestales comerciales en este país. Sin embargo, sólo en 1952 fue plantada con estos fines, obteniéndose buenos rendimientos (Albert 1908, Weber 1957). Libby (1996) planteó que es probable que la secuoya no se adapte bien a zonas sin presencia de neblina costera en verano, ya que la especie presenta un control estomático limitado en respuesta a condiciones atmosféricas de alta demanda evaporativa, no controlando bien la pérdida de agua (Burgess y Dawson 2004). Por su parte, Ahuja (1996) estableció que, en las hojas de esta especie, las células oclusivas del estoma no están bien desarrolladas para regular adecuadamente la transpiración, razón por la cual se adapta bien a las regiones costeras de California, donde la humedad y la niebla de verano son condiciones naturales, por lo que sugiere que es fundamental seleccionar procedencias resistentes a la sequía y sitios de plantación con precipitación suficiente para que la especie crezca en forma óptima. Dada la importancia que las plantaciones de secuoya pueden tener en Chile y su proyección futura (Toral et al. 2005), es interesante estudiar el proceso de aclimatación de la especie a las diferentes condiciones de plantación, para lo cual los estudios morfoanatómicos de estomas pueden contribuir a interpretar la variabilidad de respuesta de una especie introducida a las condiciones ambientales imperantes en diferentes localidades de establecimiento.

La secuoya, por tanto, es una especie de gran interés productor y presenta una de las áreas de distribución artificial más importantes entre las especies forestales, encontrándose desde China hasta el norte de Europa. Por ello, ha sido objeto de varios estudios relacionados con la variabilidad de poblaciones naturales (Noss 2000), incluyendo la plasticidad como respuesta a procesos de estrés por sequía (Burgess y Dawson 2004). Sin embargo, no hay trabajos que comparen las características morfoanatómicas de individuos de secuoya entre localidades distintas, ni tampoco en un ámbito territorial concreto, entre distintos rodales con condiciones ambientales contrastadas. En ese sentido, el objetivo de este trabajo es comparar las características morfológicas de los estomas en hojas de secuoya colectadas en cuatro plantaciones realizadas en Chile, para estudiar el grado de aclimatación de plantaciones introducidas en este país a distintas condiciones climáticas.

\section{MÉTODOS}

Procedencia del material vegetal. El material vegetal utilizado en este estudio se obtuvo en cuatro plantaciones de secuoya ubicadas en diferentes localidades de Chile, buscando un gradiente geográfico latitudinal y de condiciones ambientales. Las localidades fueron: 1) Antiquina ( $38^{\circ} 18^{\prime} \mathrm{S}, 73^{\circ} 23^{\prime}$ O, 55 m s.n.m.), situada en la región del Biobío, en un rodal de 29 años; 2) Voipir (39 $18^{\circ}$ S, $72^{\circ} 10^{\prime}$ O, 486 m s.n.m.) en la región de La Araucanía, en un rodal de 16 años de edad; 3) Lanco (39 $30^{\circ} \mathrm{S}$, $72^{\circ} 45^{\prime}$ O, 125 m s.n.m.) en la región de Los Ríos, en un rodal de 15 años; y 4) Frutillar $\left(41^{\circ} 02^{\prime} S\right.$; $73^{\circ} 02^{\prime} \mathrm{O}$, 41 m s.n.m.) en la región de Los Lagos, en un rodal de 27 años. Las localidades presentan distintas condiciones edafoclimáticas, con gradientes en la precipitación, temperaturas y altitud (cuadro 1). Los rodales se establecieron de plantas originadas de semillas procedentes del condado de Humbolt ( $40^{\circ} 01^{\prime} \mathrm{N}, 123^{\circ} 49^{\prime} \mathrm{O}$, California, EE.UU.). La superficie de rodales plantados con secuoya en Chile es escasa. Estas localidades presentaban signos de no haber sido intervenidas y los rodales estaban bien conservados por sus propietarios, con una misma densidad de plantación inicial, y sin que se hubieran realizado intervenciones silviculturales (como podas o raleos). 
Cuadro 1.Características climáticas y edáficas de las cuatro localidades de procedencia del material vegetal de Sequoia sempervirens, en Chile (CIREN, 1999ab, 2001).

Edaphic and climatic characteristics of the four locations of vegetal material of Sequoia sempervirens, in Chile (CIREN 1999ab, 2001).

\begin{tabular}{|c|c|c|c|c|}
\hline \multirow{2}{*}{ Variable } & \multicolumn{4}{|c|}{ Localidad } \\
\hline & Antiquina & Voipir & Lanco & Frutillar \\
\hline Precipitación anual (mm) & 1.261 & 2.454 & 2.139 & 1.890 \\
\hline Humedad del aire, enero ( \%) & 78 & 67 & 75 & 75 \\
\hline Temperatura máxima, enero $\left({ }^{\circ} \mathrm{C}\right)$ & 19,9 & 23,5 & 27,1 & 19,3 \\
\hline Temperatura mínima, julio $\left({ }^{\circ} \mathrm{C}\right)$ & 6,2 & 4,6 & 3,3 & 4,1 \\
\hline Período húmedo ( $\mathrm{N}^{\mathrm{o}}$ meses $)$ & 6 & 9 & 9 & 9 \\
\hline Evaporación anual (mm) & 900 & 850 & 880 & 800 \\
\hline Evaporación potencial (nov-feb) (mm) & 463,0 & 505,8 & 523,7 & 476,1 \\
\hline Profundidad (cm) & $>100$ & $75-100$ & $>100$ & $75-100$ \\
\hline & Profundo & Moderadamente profundo & Profundo & Moderadamente profundo \\
\hline Suelo Textura (horizonte 1) & Arcillosa & Franca arenosa fina & Franca arenosa & Franca \\
\hline $\mathrm{pH}$ (horizonte 1) & 5,2 & 5,9 & 6,1 & 5,6 \\
\hline Densidad aparente $\left(\mathrm{g} \mathrm{cm}^{-3}\right)$ (horizonte 1$)$ & 1,41 & 0,91 & 0,51 & 0,53 \\
\hline
\end{tabular}

Colecta de muestras y proceso de fijación de las hojas. Basado en los criterios señalados se seleccionó un rodal por localidad. En cada rodal se eligieron 10 árboles, cinco en el interior del rodal, con baja intensidad de luz, y cinco en el borde del rodal expuesto a plena insolación. En cada árbol se cortaron dos ramas de $30 \mathrm{~cm}$ de largo, del tercio inferior de la copa para facilitar la accesibilidad y selección de ramas sanas, una de exposición norte y otra de exposición sur. En las cuatro localidades se obtuvieron cinco muestras por exposición (norte-sur), dada la variabilidad interna de las hojas por la mayor o menor exposición a la luz (Ishii et al. 2008), y cinco muestras por posición del árbol dentro del rodal (interior y borde), lo que representó un total de veinte muestras de hojas por localidad, y 80 para el conjunto del estudio.

Las muestras correspondieron a hojas frescas, las cuales se guardaron en envases viales cerrados y sellados con parafina, en una solución FAA (formaldehído-ácido acético-alcohol etílico) para su fijación. La composición del fijador fue $10 \mathrm{~mL}$ de formalina $37-40 \%, 5 \mathrm{~mL}$ de ácido acético glacial 1:1, $50 \mathrm{~mL}$ de alcohol etílico $95 \%$ y $35 \mathrm{~mL}$ de agua destilada. Después de 48 horas en FAA, las hojas se cambiaron a una solución de alcohol al $70 \%$. El transporte de las hojas se realizó en un contenedor frío hasta el laboratorio y se mantuvieron en refrigeración $\left(4^{\circ} \mathrm{C}\right)$ hasta la realización de las preparaciones histológicas.

La preparación del material se hizo empleando dos técnicas. La primera de ellas consistió en la diafanización de las muestras de hojas, según el protocolo propuesto por Johansen (1940); para lo cual las hojas se sumergieron en una solución al $6 \%$ de hipoclorito de sodio, hasta alcanzar su completa decoloración. Luego las muestras se colocaron en agua destilada para proceder a separar ambas epidermis con ayuda de agujas de disección. Posteriormente las muestras se tiñeron en una solución de safranina y se montaron en portaobjetos provistos de una gota de jalea de glicerina, cubriéndose con un cubreobjetos para su posterior observación.

En la segunda técnica se procedió a deshidratar el material previamente fijado y, posteriormente, se incluyó en parafina (Johansen 1940). En el material fijado se hicieron cortes histológicos transversales de $10 \mu$ de espesor, los cuales fueron teñidos con ácido tánico (solución acuosa $1 \%$ ), cloruro férrico (solución acuosa $3 \%$ ), safranina (solución alcohólica-50 \% al $1 \%$ ) y Fast-Green® (triarilmetano en solución alcohólica-95 \% al 0,5\%). Las preparaciones se sellaron con Eukitt ${ }^{\circledR}$.

Medición del tamaño de los estomas, la densidad y el tipo y la anatomía de las células acompañantes. Las preparaciones fueron microfotografiadas con una cámara Canon acoplada a un microscopio Zeiss Axiostar Plus y el análisis de ellas se efectuó con el Programa IPLab (BD Biosciences Bioimaging, Rockville, EE.UU.).

En cada muestra se determinaron el tamaño de los estomas, el índice estomático, la longitud y el ancho de los estomas, y el tamaño de las células oclusivas y subsidiarias, obteniéndose la media de tres mediciones observadas en un área de 0,5 x 0, $4 \mathrm{~mm}$. El valor de 0,4 mm corresponde al ancho de la banda estomática en la zona central del envés de la hoja (Barrera y Mesa 1992). La densidad estomática se calculó determinando el número de estomas en la misma superficie sobre la banda estomática según el método propuesto por Dunlap y Stettler (2001) y que ha sido utilizado en otros trabajos de secuoya (Ma y Li 2002). Los diámetros mayor y menor de las células oclusivas y

\footnotetext{
$1 \quad$ Polimetacrilato de butilo O Kindler GMBH \& Co.
} 
subsidiarias, tanto izquierda como derecha, se midieron en los cortes transversales de las hojas.

Índice estomático. La determinación del índice estomático (IE) se realizó con un microscopio óptico Zeiss Axiostar Plus en un campo de 400X que cubre una superficie de $4,347 \mathrm{~mm}^{2}$. El cálculo del IE [1] se realizó a través de la ecuación sugerida por Wilkinson (1979):

Donde,

$$
I E=\left(N E^{*} 100\right) /(C E+N E)
$$

IE = Índice estomático.

$\mathrm{NE}=$ Número de estomas por campo de observación.

$\mathrm{CE}=$ Número de células epidérmicas en el campo de observación.

Análisis de datos. El análisis estadístico se inició con la comprobación de que los datos cumplían el requisito de normalidad y homogeneidad de la varianza (homocedasticidad). La normalidad se comprobó mediante la prueba de Kolmogorov-Smirnov y la homocedasticidad por la prueba de Levene. Luego se procedió a un análisis de la varianza (ANOVA) de tres factores de efectos fijos para un nivel de significación del $5 \%(P \leq 0,05)$. Cuando el análisis de la varianza fue significativo se realizó la prueba de Tukey de comparación múltiple de las medias para un nivel de significación del $5 \%(P \leq 0,05)$. Los factores analizados fueron localidad (cuatro niveles), ubicación o posición del árbol en el rodal (dos niveles) y exposición (dos niveles), con cinco repeticiones. El programa estadístico utilizado fue SPSS (Versión 12.0 1S, SPSS Inc.).

\section{RESULTADOS}

Descripción de los estomas. La hoja de secuoya tiene forma linear oblonga, coriácea, con dos bandas estomáticas en la superficie abaxial, claramente diferenciadas de las células epidérmicas por su color blanquecino. La epidermis del envés presentó en todos los casos entre tres y ocho filas de estomas a cada lado de la vena central. Los estomas, por su gran tamaño, se diferenciaron claramente por la tinción más oscura que adquieren las células oclusivas que se encuentran debajo de las células epidérmicas (figura 1A). La cavidad subestomática mostró cambios de tamaño y estructura según la posición del estoma en la hoja. En la superficie adaxial
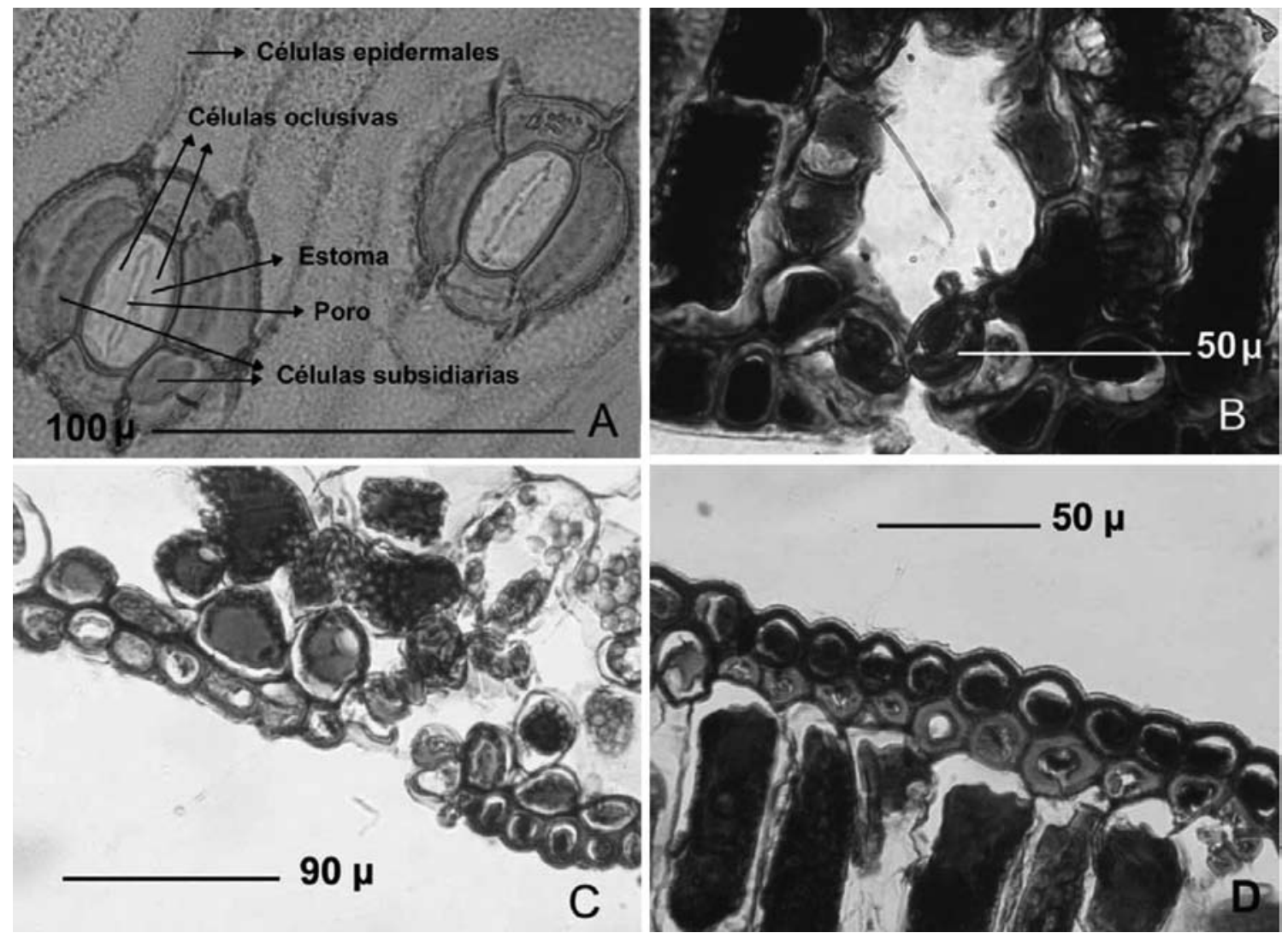

Figura 1. Estoma de Sequoia sempervirens: (A) estructura de los estomas donde se muestran las células oclusivas y subsidiarias; (B) estoma en cara adaxial; (C) estoma en cara abaxial; (D) epidermis con hipodermis esclerificada en cara adaxial.

Stomata of Sequoia sempervirens: (A) Stomata structure where guard and subsidiary cells are shown, (B) Stomata in adaxial leaf surface,

(C) Stomata in abaxial leaf surface, (D) epidermis with sclerified hypodermis in adaxial leaf surface. 
la cavidad se encuentra rodeada de células del parénquima en empalizada, siendo su forma regular y bien delimitada (figura 1B). En cambio, en la superficie abaxial la cavidad es de forma irregular ya que se encuentra circundada por células del parénquima esponjoso (figura 1C).

La hoja de secuoya posee tres canales resiníferos, siendo el central de mayor tamaño y, aparentemente, no se ven afectados por la latitud, posición en el rodal o por la exposición. En los bordes de la hoja y en la superficie adaxial, la epidermis presenta una hipodermis esclerificada, confiriéndole más rigidez y firmeza a la hoja (figura 1D). En cambio, en la superficie abaxial se observó una epidermis monoestratificada sin hipodermis con estomas solitarios o en grupos de hasta siete, de acuerdo al ancho de la banda estomática.
Densidad, índice estomático, dimensiones de los estomas y de las células oclusivas. La densidad estomática media varió entre $81,3 \pm 20,23$ estomas $\mathrm{mm}^{-2}$ y 114,4 \pm 19,9 estomas $\mathrm{mm}^{-2}$ según la localidad. Lanco presentó mayor número de estomas y difirió significativamente de Antiquina, Voipir y Frutillar, siendo estas tres últimas similares. Por otro lado, la densidad de estomas mostró diferencias en función de la ubicación del árbol en el rodal (bordeinterior), no así en el factor exposición (norte-sur). Las hojas en árboles del borde del rodal presentaron mayor densidad de estomas, en especial en la localidad de Lanco $\left(110 \pm 19,3\right.$ estomas $\mathrm{mm}^{-2}$ en el borde norte y $130 \pm 1,6$ estomas $\mathrm{mm}^{-2}$ en el borde sur, contra $108 \pm 22,3$ estomas $\mathrm{mm}^{-2}$ y $97,4 \pm 18,1$ estomas $\mathrm{mm}^{-2}$, respectivamente, en el interior) (cuadros 2 y 3 ).

Cuadro 2. Valores medios y desviaciones típicas de las variables respuestas para el factor localidad.

Mean values and standard deviations of response variables for location factor.

\begin{tabular}{|c|c|c|c|c|c|}
\hline \multirow{2}{*}{ Variable } & \multicolumn{4}{|c|}{ Localidades } & \multirow{2}{*}{$\begin{array}{c}\text { Significancia* } \\
\quad P(3,64)\end{array}$} \\
\hline & Antiquina & Voipir & Lanco & Frutillar & \\
\hline Densidad estomática (número $\mathrm{mm}^{-2}$ ) & $81,3 \pm 20,23 a$ & $82,7 \pm 21,9 a$ & $111,4 \pm 19,9 b$ & $86,5 \pm 19,8 \mathrm{a}$ & $<0,001$ \\
\hline Índice estomático & $21,1 \pm 4,8 \mathrm{a}$ & $18,6 \pm 2,4 \mathrm{ab}$ & $15,5 \pm 1,7 b$ & $16,3 \pm 1,9 b$ & $<0,001$ \\
\hline Longitud de estomas $(\mu \mathrm{m})$ & $31,4 \pm 7,2$ & $34,8 \pm 4,1$ & $33,9 \pm 4,2$ & $37,7 \pm 14,2$ & 0,093 \\
\hline Ancho de estomas $(\mu \mathrm{m})$ & $12,7 \pm 2,9 a$ & $12,6 \pm 2,1 \mathrm{ab}$ & $14,8 \pm 2,2 b c$ & $14,9 \pm 3,3 c$ & 0,004 \\
\hline Ancho de banda estomática $(\mu \mathrm{m})$ & $0,72 \pm 0,1$ & $0,65 \pm 0,1$ & $0,73 \pm 0,2$ & $0,75 \pm 0,1$ & 0,189 \\
\hline Ancho célula oclusiva derecha $(\mu \mathrm{m})$ & $9,5 \pm 2,1$ & $9,0 \pm 1,8$ & $9,1 \pm 1,9$ & $9,2 \pm 1,7$ & 0,892 \\
\hline Largo célula oclusiva derecha $(\mu \mathrm{m})$ & $17,0 \pm 3,5$ & $18,7 \pm 3,5$ & $18,4 \pm 3,1$ & $17,9 \pm 4,2$ & 0,480 \\
\hline Ancho célula oclusiva izquierda $(\mu \mathrm{m})$ & $8,7 \pm 2,1$ & $9,7 \pm 1,8$ & $9,6 \pm 1,6$ & $9,1 \pm 1,8$ & 0,274 \\
\hline Largo célula oclusiva izquierda $(\mu \mathrm{m})$ & $17,8 \pm 4,7$ & $20,4 \pm 3,3$ & $18,9 \pm 2,2$ & $18,1 \pm 3,1$ & 0,087 \\
\hline Ancho célula subsidiaria derecha $(\mu \mathrm{m})$ & $10,4 \pm 3,1$ & $11,4 \pm 2,5$ & $11,4 \pm 3,7$ & $9,6 \pm 2,4$ & 0,165 \\
\hline Largo célula subsidiaria derecha $(\mu \mathrm{m})$ & $22,9 \pm 5,3$ & $23,9 \pm 3,1$ & $23,2 \pm 3,9$ & $23,6 \pm 4,5$ & 0,897 \\
\hline Ancho célula subsidiaria izquierda $(\mu \mathrm{m})$ & $10,1 \pm 2,4$ & $11,2 \pm 1,9$ & $10,2 \pm 2,4$ & $10,0 \pm 2,2$ & 0,331 \\
\hline Largo célula subsidiaria izquierda $(\mu \mathrm{m})$ & $21,8 \pm 2,9$ & $22,9 \pm 2,9$ & $24,2 \pm 4,0$ & $23,9 \pm 4,5$ & 0,156 \\
\hline
\end{tabular}

* Localidades con diferente letra presentan diferencias significativas $(P \leq 0,05)$.

Cuadro 3. Valores medios y desviación típica de las variables de respuestas para el factor exposición y ubicación en el rodal de Sequoia sempervirens.

Mean values and standard deviation of response variables for slope exposition and location in the forest stand of Sequoia sempervirens.

\begin{tabular}{|c|c|c|c|c|c|c|}
\hline \multirow[b]{2}{*}{ Variable } & \multicolumn{3}{|c|}{ Exposición } & \multicolumn{3}{|c|}{ Ubicación en el rodal } \\
\hline & Norte & Sur & $\begin{array}{c}\text { Significancia } \\
P(1,64)\end{array}$ & Borde & Interior & $\begin{array}{c}\text { Significancia } \\
P(1,64)\end{array}$ \\
\hline Densidad estomática (número/mm²) & $91,1 \pm 23,7$ & $89,8 \pm 23,6$ & 0,770 & $96,5 \pm 24,0$ & $84,3 \pm 21,6$ & $0,008 *$ \\
\hline Índice estomático & $18,1 \pm 2,6$ & $18,6 \pm 4,1$ & 0,420 & $19,2 \pm 3,8$ & $17,6 \pm 2,8$ & $0,010 *$ \\
\hline Longitud de estomas $(\mu \mathrm{m})$ & $33,4 \pm 3,9$ & $35,6 \pm 11,5$ & 0,229 & $34,3 \pm 11,3$ & $34,6 \pm 4,7$ & 0,878 \\
\hline Ancho de estomas $(\mu \mathrm{m})$ & $13,8 \pm 2,6$ & $13,7 \pm 3,2$ & 0,949 & $14,2 \pm 2,9$ & $13,3 \pm 2,7$ & 0,118 \\
\hline Ancho de banda estomática ( $\mu \mathrm{m})$ & $0,71 \pm 0,12$ & $0,71 \pm 0,17$ & 0,941 & $0,72 \pm 0,14$ & $0,71 \pm 0,15$ & 0,710 \\
\hline Ancho célula oclusiva derecha $(\mu \mathrm{m})$ & $9,23 \pm 1,8$ & $9,22 \pm 1,92$ & 0,995 & $9,29 \pm 1,92$ & $9,16 \pm 1,81$ & 0,771 \\
\hline Largo célula oclusiva derecha $(\mu \mathrm{m})$ & $17,6 \pm 3,1$ & $18,4 \pm 3,9$ & 0,320 & $17,8 \pm 2,1$ & $18,3 \pm 4,6$ & 0,730 \\
\hline Ancho célula oclusiva izquierda ( $\mu \mathrm{m})$ & $9,3 \pm 2,1$ & $9,3 \pm 1,6$ & 0,980 & $8,9 \pm 1,8$ & $9,6 \pm 1,8$ & 0,140 \\
\hline Largo célula oclusiva izquierda ( $\mu \mathrm{m})$ & $18,8 \pm 2,9$ & $18,2 \pm 4,0$ & 0,997 & $19,4 \pm 3,5$ & $18,2 \pm 3,5$ & 0,123 \\
\hline Ancho célula subsidiaria derecha ( $\mu \mathrm{m})$ & $10,5 \pm 3,2$ & $10,8 \pm 2,8$ & 0,717 & $9,8 \pm 2,9$ & $11,4 \pm 2,9$ & $0,020 *$ \\
\hline Largo célula subsidiaria derecha $(\mu \mathrm{m})$ & $23,1 \pm 4,3$ & $23,6 \pm 4,1$ & 0,643 & $22,4 \pm 4,6$ & $24,4 \pm 3,6$ & 0,054 \\
\hline Largo célula subsidiaria izquierda $(\mu \mathrm{m})$ & $23,3 \pm 3,2$ & $23,2 \pm 4,1$ & 0,914 & $22,8 \pm 3,5$ & $23,6 \pm 3,9$ & 0,326 \\
\hline
\end{tabular}

* Significativo para $P \leq 0,05$ según factor. 
El índice estomático se encontró entre 15,5 $\pm 1,7$ y $21,1 \pm 4,8$ según la localidad. Antiquina tuvo un mayor índice estomático y difirió significativamente de Voipir, Lanco y Frutillar (cuadro 2). Así también Voipir difirió de Lanco y Frutillar; por otro lado, Lanco y Frutillar fueron similares. El índice estomático mostró efectos significativos del factor ubicación del árbol en el rodal (borde-interior), no así del factor exposición (norte-sur). Las hojas obtenidas de árboles ubicados en el borde del rodal presentaron un mayor índice estomático, particularmente en la localidad Antiquina $(20,4 \pm 2,6$ borde norte y $23,9 \pm 2,6$ borde sur (cuadro 3).

La longitud media de los estomas varió entre $31,4 \pm 7,2$ y $37,7 \pm 14,2 \mu \mathrm{m}$ y su ancho medio entre $12,6 \pm 2,1$ y $14,8 \pm 2,2 \mu \mathrm{m}$. La longitud del estoma no presentó diferencias significativas, sí en su ancho medio, siendo todas las localidades diferentes entre sí (cuadros 2 y 3).

Las variables densidad, índice estomático, longitud y ancho de los estomas presentaron diferencias en los factores principales señalados, no así en sus interacciones. El resto de las variables analizadas (cuadro 3) no presentó diferencias estadísticas entre los factores principales de localidad, exposición y ubicación en el rodal, como tampoco en las interacciones.

\section{DISCUSIÓN}

Los estomas son las estructuras de las hojas que más contribuyen en el control del intercambio de gases $\left(\mathrm{CO}_{2} \mathrm{y}\right.$ agua) en las plantas (Willmer y Fricket 1996). Los factores ambientales, tales como la intensidad de la luz, la concentración atmosférica de $\mathrm{CO}_{2}$ y los sistemas internos de control regulan el desarrollo de los estomas (Hetherington y Woodward 2003). La conductancia hidráulica de la planta, desde el suelo hasta la hoja, y la respuesta de los estomas a los cambios en las condiciones ambientales son características principales que regulan el proceso de transpiración (Raven 2002). En este trabajo se ha estudiado si las características morfológicas de los estomas de secuoya han respondido de manera diferencial a las condiciones ambientales en cuatro localizaciones en Chile.

Los estomas de la epidermis abaxial en las poblaciones estudiadas en Chile presentan diferencias morfológicas en algunos parámetros cuando se comparan con los datos obtenidos para la especie en California. La longitud media de los estomas y su ancho medio medidos en Chile indican valores próximos al límite inferior para la especie en el caso de la longitud, y por debajo de los valores mínimos para la anchura al compararlos con los observados para la especie en su área de distribución natural (longitud 25-150 $\mu \mathrm{m}$, ancho 15-37,5 $\mu \mathrm{m}$, Ma y Li 2002). La especie en Chile tiene estomas algo más pequeños, modificando la resistencia estomática a la transpiración (y por tanto la transpiración), lo que puede condicionar la productividad al estar ésta relacionada con el consumo y disponibilidad de agua. El tamaño de los estomas es un factor clave en el proceso de aclimatación al estrés hídrico, al existir una relación inversa entre el tamaño del estoma y la resistencia a la sequía (Strasburger et al. 1986, Aasaman et al. 2001). El número de células subsidiarias (4-6) para el conjunto de las poblaciones, coincidió con los valores obtenidos en otros trabajos (Srinivasan y Friis 1989, Chaturvedi 1993, Villar de Seoane 1998, Ma y Li 2002). Sin embargo, los valores de la longitud $(18,4 \mu \mathrm{m})$ y del ancho $(9,2 \mu \mathrm{m})$ de las células oclusivas también mostraron valores inferiores a los observados por Ma y Li (2002) (35-70 $\mu \mathrm{m}$ de longitud y $7-20 \mu \mathrm{m}$ de ancho), en particular para la longitud, lo que viene a confirmar un cierto grado de aclimatación del aparato estomático a condiciones de mayor estrés (Takur 1990).

En cuanto a la densidad y el índice estomático, los valores obtenidos en Chile (DE de 90 estomas $\mathrm{mm}^{-2}$, IE: 18,4) también mostraron diferencias con respecto a California (DE: $128 \mathrm{~mm}^{-2}$, IE: 13,8). La disminución de la densidad estomática de los árboles en Chile incrementaría la resistencia estomática y evitaría el exceso de transpiración (Takur 1990), posibilitando una mejor adaptación de los árboles a condiciones de mayor demanda hídrica. Respecto al IE, aunque algunos trabajos mencionan que se mantiene sin cambios como respuesta a las condiciones ambientales (Wilkinson 1979), en trabajos posteriores se ha observado que es una variable muy sensible a condiciones ambientales (luz, agua, nutrientes) estresantes (Kürschner et al. 1998). En el caso de los árboles estudiados en Chile se observó una baja densidad de estomas y un valor alto del índice estomático, relacionado con un aumento del tamaño de las células epidérmicas. Este tamaño grande de las células epidérmicas (que reduce el denominador de la fórmula de IE por unidad de superficie analizada) posiblemente estaría relacionado con la optimización de la capacidad de almacenar agua (Strasburger et al. 1986). Todos estos valores indican cambios en la regulación estomática que podrían relacionarse con una menor disponibilidad de humedad atmosférica debida a la ausencia de criptoprecipitación en las localizaciones chilenas, frente a las poblaciones naturales (Burgess y Dawson 2004).

Al comparar las características de los estomas entre las localidades de Chile se observó sólo una tendencia similar. Los estomas mostraron una propensión a un mayor tamaño a mayor latitud y mayor precipitación (Antiquina: $31,4 \mu \mathrm{m}$ frente a Voipir: $34,8 \mu \mathrm{m}$, Lanco $33,9 \mu \mathrm{m}$ y Frutillar $37,7 \mu \mathrm{m}$ ), aunque las diferencias no fueron significativas (cuadro 2). El valor medio de la densidad de estomas para el conjunto de las localidades fue de 90,1 estomas $\mathrm{mm}^{-2}$, con valores medios por localidad significativamente diferentes entre la localidad de Lanco $\left(111,4\right.$ estomas $\left.\mathrm{mm}^{-2}\right)$ y el resto de los lugares de plantación $\left(<87\right.$ estomas $\mathrm{mm}^{-2}$ ). El índice estomático tuvo un comportamiento similar a la densidad, siendo las diferencias significativas entre Antiquina $(21,1)$ y el resto de los lugares de plantación. Los resultados obtenidos parecen confirmar, aunque de 
forma menos clara cuando se comparan las poblaciones chilenas y californianas, un cierto grado de aclimatación de las poblaciones en Chile en función de las condiciones ambientales. El tamaño de los estomas, la densidad y el índice estomático varían en función de la latitud, que pueden venir asociados a cambios en el fotoperíodo, la disponibilidad hídrica y las condiciones edáficas. La población de Antiquina, la más norteña, presenta la precipitación más baja y los suelos de mayor densidad aparente, lo que puede relacionarse con un mayor nivel de estrés y una mayor adecuación del aparato estomático a esas condiciones. La población de Voipir presentó valores similares a Antiquina, siendo la población de Lanco la que mostró valores más próximos a los de referencia para la especie (Ma y Li 2002), al vegetar en un clima húmedo y templado y sobre un suelo de buena calidad (profundo y de textura franca arenosa), lo que hace de esta localidad la menos estresante de todas las estudiadas. En el caso de Frutillar, las condiciones también mostraron un gradiente de adaptación a los niveles de estrés hídrico, con estomas grandes, pero con densidad e índice estomático menores que Lanco, al tener una precipitación menor y un suelo de peor calidad (cuadro 1).

La morfología de los estomas juega un papel importante en el control de la pérdida de agua, lo cual permite adaptarse a muchas especies vegetales a condiciones ambientales muy diversas (Hetherington y Woodward 2003). La variación de las características de los estomas podría desempeñar un papel importante en el proceso de aclimatación de una especie al ambiente. La secuoya en su área de distribución natural está principalmente limitada por factores climáticos (Dawson 1998), en particular por la presencia de criptoprecipitación estival, que puede llegar a aportar hasta el $40 \%$ de la precipitación total durante dicho período (Dawson 1998, Burgess y Dawson 2004). Las plantaciones establecidas en Chile no presentan estos fenómenos de precipitación horizontal, lo que podría hacer pensar, de acuerdo a la hipótesis de Libby (1996) y Ahuja (1996), que la secuoya no se adaptaría adecuadamente a zonas sin presencia de neblina costera en verano, por limitaciones relacionadas con la morfología de sus estomas. En este trabajo se muestra que la especie adecua su aparato estomático a las condiciones generales de mayor sequía en las localidades chilenas si se comparan con los valores de referencia para la especie en California (Ma y Li 2002). El tamaño de los estomas y la densidad parecen ser las variables más sensibles al cambio en las condiciones ambientales, posiblemente relacionadas con la resistencia estomática, lo que evitaría el exceso de transpiración y permitiría una mejor adaptación de los árboles a condiciones de mayor demanda hídrica (Strasburger et al. 1986, Aasaman et al. 2001). Cuando se comparan las localidades en Chile, si bien se obtuvieron diferencias significativas para algunas variables entre localidades, los cambios en el aparato estomático no fueron tan marcados en respuesta a las características ambientales de los sitios. Sin embargo, los resultados muestran que aquellas localidades con condiciones climáticas más limitantes (menor humedad atmosférica y precipitación), en combinación con peores condiciones edáficas (suelos con mayor densidad aparente y menor profundidad), tuvieron estomas más pequeños y en menor densidad, mostrando un importante grado de plasticidad fenotípica de la especie para el carácter evaluado, que permite procesos de aclimatación a situaciones ambientales contrastantes. Por otro lado, si bien los sitios difieren en precipitaciones medias anuales, la demanda evaporativa del verano es similar entre los mismos, por lo que parece que las condiciones edáficas relacionadas con la disponibilidad de agua en el suelo (profundidad y textura) pudieran ser más determinantes en los niveles de estrés hídrico y, por tanto, de aclimatación de la especie en estas localidades. Sobre la base de estos resultados, sería posible particularizar las zonas de mayor potencial para el establecimiento de la especie en Chile a partir de los modelos generales propuestos (Toral et al. 2005).

\section{AGRADECIMIENTOS}

Se agradece a Forestal Simpson-Chile y especialmente a JA Rydelius, J Ross y S Valdés por el aporte de la colección Kuser a la Universidad de Chile, y al Fondo Nacional de Desarrollo Científico y Tecnológico (Proyecto FONDEF DO1 I 1008 - CHILE), que financió el desarrollo de esta investigación. Al mismo tiempo se agradece a Forestal Voipir, Forestal Natalhue e Instituto Forestal por su apoyo al desarrollo de este trabajo.

\section{REFERENCIAS}

Aasaman K, A Sober, M Rahi. 2001. Leaf anatomical characteristic associated with shoot hydraulic conductance, stomatal conductance and stomatal sensitivity to changes of leaf water status in temperate deciduous trees. Aust. J. Plant Physiol. 28: 765-774.

Ahuja M. 1996. Micropropagation and field testing of frosttolerant Sequoia sempervirens genotypes. In Conference on Coast Redwood Forest Ecology \& Management. June 18-20. Consultado 10 ago. 2009. Disponible en http://www.cnr.berkeley. edu/ jleblanc/WWW/ Redwood/rdwd-Micropro.html

Albert F. 1908. Los siete árboles forestales más recomendables para el país. In Algunas observaciones sobre las especies en la sección de aguas y bosque. Santiago, Chile. Cervantes. 52 p.

Barrera E, I Mesa. 1992. Características de la epidermis foliar de árboles chilenos. I. Subclase Magnoliidae. Chile. Boletín del Museo Nacional de Historia Natural 43: 29-39.

Benson L. 1979. Plant Classification, $2^{\text {nd }}$ ed. Heath DC, Lexington, MA, USA. p. 227-229.

Burgess S, T Dawson. 2004. The contribution of fog to the water relations of Sequoia sempervirens (D. Don): foliar uptake and prevention of dehydration. Plant, Cell and Environment 27: 1023-1034. 
Chaturvedi S. 1993. Morphological, cuticular and anatomical studies of some members of Taxodiaceae. Bionature 13: 127-131.

CIREN (Centro de Investigación de Recursos Naturales, CL). 1999a. Estudio Agrológico VIII Región. Descripciones de Suelos, Materiales y Símbolos. Publicación No 121. Tomos I y II. Santiago, Chile. CIREN. 586 p.

CIREN (Centro de Investigación de Recursos Naturales, CL). 1999b. Estudio Agrológico IX Región. Descripciones de Suelos, Materiales y Símbolos. Publicación No 122. Santiago, Chile. CIREN. 326 p.

CIREN (Centro de Investigación de Recursos Naturales, CL). 2001. Estudio Agrológico X Región. Descripciones de Suelos, Materiales y Símbolos. Tomos I y II. Santiago, Chile. CIREN. 412 p.

Dawson T. 1998. Fog in the California redwood forest: ecosystem inputs and use by plants. Oecologia 117: 476-485.

DeLucia EH, H Maherali, EV Carey. 2000. Climate-driven changes in biomass allocation in pines. Global Change Biol. 6: 587-593.

Dunlap J, R Stettler. 2001. Variation in leaf epidermal and stomatal traits of Populus trichocarpa from two transects across the Washington Cascades. Canadian Journal of Botany 79 (5): 528-535.

Hetherington AM, FI Woodward. 2003. The role of stomata in sensing and driving environmental change. Nature 21: 901-908.

Ishii HT, GM Jennings, SC Sillett, GW Koch. 2008. Hydrostatic constraints on morphological exploitation of light in tall Sequoia sempervirens trees. Oecologia 156: 751-763.

Johansen R. 1940. Plant microtechnique. New York, USA. McGraw-Hill. 523 p.

Kürschner W, I Stulen, F Wagner, P Kuiper. 1998 Comparison of palaeobotanical observations with experimental data on the leaf anatomy of durmast oak (Quercus petraea) (Fagaceae) in response to environmental changes. Annals of Botany 81: 657-664.

Libby W. 1996. Ecology and management of Coast Redwood. In Conference on Coast Redwood Forest Ecology \& Management. June 18-20. Consultado 17 oct. 2008. Disponible en http://www.cnr.berkeley.edu/ jleblanc/WWW/Redwood/ rdwd-Micropro.html

Ma QW, CS Li. 2002. Epidermal structures of Sequoia sempervirens (D. Don) Endl. (Taxodiaceae). Taiwania 47(3): 194-202.

Ma QW, FL Li, CS Li. 2005. The coast redwoods (Sequoia, Taxodiaceae) from the Eocene of Heilongjiang and the
Miocene of Yunnan, China. Review of Palaeobotany and Palynology 135(3/4): 117-129.

Mencuccini M. 2003. The ecological significance of long-distance water transport: short-term regulation, long-term acclimation and the hydraulic costs of stature across plant life forms. Plant Cell Environ 26: 163-182.

Mencuccini M, L Bonosi. 2001. Leaf/sapwood area ratios in Scots pine show acclimation across Europe. Can. J. For. Res. 31: 442-456.

Noss RF. 2000. The redwood forest: history, ecology and conservation of the Coastal California Redwoods. Covelo, EE.UU. Island Press. 335 p.

Poyatos R, J Martínez-Vilalta, J Cermák, R Ceulemans, A Granier, J Irvine, B Köstner, F Lagergren, L Meiresonne, N Nadezhdina, R Zimmermann, P Llorens, M Mencuccini. 2007. Plasticity in hydraulic architecture of Scots pine across Eurasia. Oecologia 153: 245-259.

Raven J. 2002. Selection pressures on stomatal evolution. New Phytol. 153: 371-386.

Srinivasan V, E Friis. 1989. Taxodiaceous conifers from the upper cretaceous of Sweden. Bio. Skifter 35: 5-57.

Strasburger E, F Noll, H Schenck, A Schimper. 1986. Tratado de Botánica. Barcelona, España, EM Marin. 1098 p.

Takur P. 1990. Different physiological response of tomato (Lycopersicum esculentum Mill.) cultivar to drought. Acta Physiologiae Plantarum 12: 175-182.

Toral Ibánez M, L González, R Garfias (eds.). 2005. Secuoya: nueva opción, productos y mercados para el sur de Chile. Santiago de Chile, Universidad de Chile, Facultad de Ciencias Forestales. 208 p.

Trewavas A. 2003. Aspects of Plant Intelligence. Annals of Botany 92: 1-20.

Villar de Seoane L. 1998. Comparative study of extend and fossil conifer leaves from the Baquero Formation (Lower Cretaceous), Santa Cruz Province, Argentina. Rev. Palaeobot. Palynol. 99: 247-263.

Weber W. 1957. Silvicultura chilena actual. Santiago, Chile. Ed. Hucke. 75 p.

Wilkinson H. 1979. The plant surface (mainly leaf). In Metcalfe CR y L Chalk eds. Anatomy of Dicotyledons. Oxford, London, UK. Claredon Press. p. 97-165.

Willmer CM, M Fricket. 1996. Stomata. $2^{\text {nd. }}$ ed. London, UK. Chapman \& Hall. 375 p.

Recibido: 11.05 .09

Aceptado: 30.04 .10 